\title{
TIN VITRO BREAST CANCER CHEMORESISTANCE TEST
}

Martina Lichtenfels', Camila Alves Silva', Caroline Brunetto Farias ${ }^{1}$ Alessandra Borba Anton Souza², Antônio Luiz Frasson²

'Ziel Biosciences Pesquisa, Desenvolvimento e Diagnóstico LTDA - Porto Alegre (RS), Brazil.

${ }^{2}$ Breast Cancer Center São Lucas Hospital, Pontificia Universidade Católica - Porto Alegre (RS), Brazil.

Introduction: Tumor resistance is the main cause of treatment failure leading to cancer progression and is classified into intrinsic and acquired resistance. Intrinsic resistance is related to a preexisting condition and acquired resistance is induced by a drug. Some methods are already available worldwide to assess drug resistance, however, in Brazil no in vitro chemoresistance test for cancer is validated for clinic use. Objectives: The aim of our study was to validate the in vitro chemoresistance test Chemobiogram for the drugs used in breast cancer (BC) treatment. An incomplete response to neoadjuvant treatment was used to validate the results at a short-term follow-up and treatment after primary BC will be used to validate the test in a long-term follow-up. Methods: Patients with invasive breast cancer were included in this initial report. Fresh tumor samples were collected during surgery and subsequently dissociated to obtain tumor cells. The tumor cells were cultured in a 96 well plate with the several drugs used for BC treatment, including cytotoxic, hormonal, antiHER2, and target therapies, and after 72 hours, cell viability was evaluated. The test result is defined based on cell viability as low $(<40 \%)$, medium (40\%-60\%), and high $(>60 \%)$ resistance. The test result is compared to the patient's response to the treatment. Results: To validate the dissociation and BC primary culture techniques we collected samples from six patients with in situ and invasive tumors. These samples were not tested in Chemobiogram. Samples from five BC patients undergoing neoadjuvant treatment and from three patients with primary $\mathrm{BC}$ were tested in the Chemobiogram. Of the five patients who underwent neoadjuvant treatment, two performed hormone therapy and three underwent chemotherapy. Four patients presented incomplete response to the treatment and one patient who underwent neoadjuvant chemotherapy presented disease progression during treatment. The chemoresistance test was able to demonstrate medium to high resistance for the drugs used in the neoadjuvant treatments (acquired resistance). The three patients with primary $\mathrm{BC}$ were diagnosed with Luminal tumors-HER2 negative. In the chemoresistance test all samples presented medium to high resistance to anti-HER2 drugs (intrinsic resistance) and low to medium resistance to cytotoxic drugs. These patients will be followed in the long term to compare patient outcomes with the test results. Conclusions: The primary culture of breast tumors was efficiently established and the preliminary result of the chemoresistance test was in accordance with the outcomes from five patients who underwent neoadjuvant treatment. This preliminary finding showed the capacity of the Chemobriogram to demonstrate drug resistance in accordance with the clinic and highlighted the importance of the in vitro chemoresistance test to avoid the use of inefficient drugs, improving and personalizing breast cancer treatment. 